\title{
TIME DISCRETIZATION OF PIECEWISE AFFINE SYSTEMS WITH SLIDING MODES
}

\author{
Michael Schwarz* Uwe Kiencke* \\ Thomas Erhard Hodrus ${ }^{* *}$ Volker Krebs ${ }^{* *}$ \\ * Institute of Industrial Information Technology, \\ ** Control Systems Laboratory, \\ University of Karlsruhe, \\ phone: +49 721 608-4515, \\ email: $\{$ schwarz, kiencke\}@iiit.uni-karlsruhe.de
}

\begin{abstract}
Piecewise affine systems are systems with a piecewise defined state equation. The continuous dynamics of these systems are affine but switch at the boundaries of certain subsets of the state-space. At these boundaries sliding modes may occur. A method will be presented to obtain a discrete-time system from a given continuous-time piecewise affine system. This method takes into account sliding modes as well as changes of the dynamics which occur between two sampling instances. Even for low sampling rates the resulting model shows a high accuracy. Copyright $\left.{ }^{(}\right) 2005$ IFAC
\end{abstract}

Keywords: Discrete-time System, Hybrid System, Nonlinear System, Piecewise Affine System, Sliding Mode

\section{INTRODUCTION}

Hybrid systems are dynamic systems which consist of a time-driven and an event-driven component. Their interaction leads to the hybrid phenomena formulated in (Branicky, M. S., 1995). A promising approach for system modelling and controller design uses Mixed Logical Dynamical models (MLD) for a predictive controller design. This approach is based on discrete-time piecewise affine systems (Bemporad, A. and Morari, M., 1999) which can be obtained via identification of the hybrid system (Münz, E. and Krebs, V., 2002) or just by theoretical modelling.

The conventional method to map a continuoustime piecewise affine system to a discrete-time piecewise affine system is to discretize the model on each domain. For applications with low sampling frequencies this approach is not feasible. A more accurate method which considers events be- tween two sampling instances has been introduced in (Hodrus, T. E. et al., 2004). The aim of this paper is to show how this method can be applied to more complex systems and how to deal with sliding modes.

Section 2 gives a definition of piecewise affine systems and an approach to model sliding modes. In section 3 the time-discretization method is described. With an example the method is demonstrated in section 4 . The contribution is finished with a short conclusion.

\section{MODELLING OF PIECEWISE AFFINE SYSTEMS}

Piecewise affine systems represent a class of hybrid systems as their dynamics may switch. Such systems can be described with the net state model (Nenninger, G. M., 2001; Schnabel, M., 2001). It 
consists of an interpreted petri net, an extended state space model and two interfaces to model the interaction of the event-driven and the timedriven component. The basic notations of the net state model are used for the following considerations.

Definition 2.1 (Piecewise affine system)

$A$ piecewise affine system is a dynamic system with the state equation

$$
\dot{\boldsymbol{x}}(t)=\left\{\begin{array}{lc}
\boldsymbol{A}_{1} \boldsymbol{x}(t)+\boldsymbol{B}_{1} \boldsymbol{u}(t)+\boldsymbol{a}_{1} & \text { if } \boldsymbol{z} \in \Omega_{1} \\
\vdots & \vdots \\
\boldsymbol{A}_{K} \boldsymbol{x}(t)+\boldsymbol{B}_{K} \boldsymbol{u}(t)+\boldsymbol{a}_{K} & \text { if } \boldsymbol{z} \in \Omega_{K}
\end{array}\right.
$$

where $\boldsymbol{z}=[\boldsymbol{x}, \boldsymbol{u}]^{T}$ is the extended state vector. The so-called trigger sets $\Omega_{i}$ are disjunctive convex polyhedra in the extended state space $\mathcal{Z}:=$ $\mathcal{X} \times \mathcal{U}$ bounded by $R\left(\Omega_{i}\right)$ hyperplanes:

$$
\begin{gathered}
\Omega_{i}:=\left\{\boldsymbol{z} \mid \forall r \in\left[1, R\left(\Omega_{i}\right)\right]: \boldsymbol{\omega}_{i r}^{T} \boldsymbol{z}-\omega_{0} \text { ir }>0\right\} \\
\Omega_{i} \cap \Omega_{j}=\varnothing \quad \text { if } i \neq j
\end{gathered}
$$

When the extended state space vector enters a new trigger set, the system dynamic changes. Adopting the mindset of the net state model, each trigger set $\Omega_{i}$ can be assigned to a discrete state $\boldsymbol{x}_{D i}$ of the system: $\boldsymbol{z} \in \Omega_{i} \Leftrightarrow \boldsymbol{x}_{D}=\boldsymbol{x}_{D i}$

The union of the trigger set $\Omega_{i}$ and its boundary $\partial \Omega_{i}$ is called the neutral set $\Gamma\left(\boldsymbol{x}_{D i}\right)=\Omega_{i} \cup \partial \Omega_{i}$ of the discrete state $\boldsymbol{x}_{D i}$. Trigger sets which cause a change of the discrete state are called active trigger sets $\Omega_{j}^{*}\left(\boldsymbol{x}_{D i}\right)$.

The previous considerations disregard the behaviour of the system at the boundaries of active trigger sets $\partial \Omega_{j}^{*}\left(\boldsymbol{x}_{D i}\right)$. It is discussed in (Hodrus, T. E. et al., 2004).

In the sequel

$$
\begin{aligned}
\partial \omega_{j}^{*}\left(\boldsymbol{x}_{D i}\right):=\partial \Omega_{i} \cap \partial \Omega_{j}^{*}\left(\boldsymbol{x}_{D i}\right) \\
\subseteq\left\{\boldsymbol{z} \mid \boldsymbol{\omega}_{j}^{* \mathrm{~T}} \boldsymbol{z}-\omega_{0 j}^{*}=0\right\}
\end{aligned}
$$

is called the active boundary of the active trigger set $\Omega_{j}^{*}\left(\boldsymbol{x}_{D i}\right)$. The system will enter the active trigger set $\Omega_{j}^{*}\left(\boldsymbol{x}_{D i}\right)$ if $\boldsymbol{z}(t) \in \partial \omega_{j}^{*}\left(\boldsymbol{x}_{D i}\right)$ holds and the condition of transition $\boldsymbol{\omega}_{j}^{* \mathrm{~T}} \dot{\boldsymbol{z}}(t)>0$ is met. The intention of this article is a discrete-time description of piecewise affine systems. Therefore, it can be assumed that the input vector $\boldsymbol{u}(t)$ is constant between two sampling instances. With $\boldsymbol{\omega}_{j}^{* \mathrm{~T}}=\left[\boldsymbol{\omega}_{x j}^{* \mathrm{~T}}, \boldsymbol{\omega}_{u j}^{* \mathrm{~T}}\right]$ the condition of transition yields

$$
\left[\boldsymbol{\omega}_{x j}^{* \mathrm{~T}} \boldsymbol{A}_{i}, \boldsymbol{\omega}_{x j}^{* \mathrm{~T}} \boldsymbol{B}_{i}\right] \boldsymbol{z}(t)+\boldsymbol{\omega}_{x j}^{* \mathrm{~T}} \boldsymbol{a}_{i}>0 .
$$

The subset of $\partial \omega_{j}^{*}\left(\boldsymbol{x}_{D i}\right)$ which meets the condition of transition is called transition set $\Xi_{j}^{*}\left(\boldsymbol{x}_{D i}\right)$.

A sliding set is a non-empty set $\Pi_{m}^{*}:=\Xi_{j}^{*}\left(\boldsymbol{x}_{D i}\right) \cap$ $\Xi_{i}^{*}\left(\boldsymbol{x}_{D j}\right)$. If such a sliding set exists the transition sets are redefined as

$$
\begin{aligned}
& \Xi_{j}^{* \text { new }}\left(\boldsymbol{x}_{D i}\right):=\Xi_{j}^{*}\left(\boldsymbol{x}_{D i}\right) \backslash \Pi_{m}^{*} \text { and } \\
& \Xi_{i}^{* \text { new }}\left(\boldsymbol{x}_{D j}\right):=\Xi_{i}^{*}\left(\boldsymbol{x}_{D j}\right) \backslash \Pi_{m}^{*} .
\end{aligned}
$$

When the extended state vector $\boldsymbol{z}(t)$ enters the sliding set $\Pi_{m}^{*}$ the system will toggle infinitely fast between the discrete states $\boldsymbol{x}_{D i}$ and $\boldsymbol{x}_{D j}$. In order to obtain a defined behaviour for this case FilipPov's method (Nenninger, G. M., 2001; Utkin, V. I., 1992) can be applied: A new discrete state $\boldsymbol{x}_{D m}$ is introduced which is assigned to the sliding set $\Pi_{m}$. When $\boldsymbol{z}(t)$ enters $\Pi_{m}$ the system changes to the sliding state $\boldsymbol{x}_{D m}$. The system dynamic in the sliding state is a weighted average of the dynamics $\dot{\boldsymbol{x}}_{i}$ and $\dot{\boldsymbol{x}}_{j}$ assigned to the corresponding discrete states $\boldsymbol{x}_{D i}$ and $\boldsymbol{x}_{D j}$ :

$$
\dot{\boldsymbol{x}}=\mu \dot{\boldsymbol{x}}_{i}+(1-\mu) \dot{\boldsymbol{x}}_{j}
$$

with $\dot{\boldsymbol{x}}_{i / j}=\boldsymbol{A}_{i / j} \boldsymbol{x}+\boldsymbol{B}_{i / j} \boldsymbol{u}+\boldsymbol{a}_{i / j}$. The sliding set $\Pi_{m}$ is a subset of a hyperplane in the extended state space with the normal vector $\boldsymbol{\pi}_{m}=$ $\left[\boldsymbol{\pi}_{x m}^{\mathrm{T}}, \boldsymbol{\pi}_{u m}^{\mathrm{T}}\right]^{\mathrm{T}}$. The parameter $\mu$ is determined by eq. (2) and the sliding condition

$$
\boldsymbol{\pi}_{x m}^{\mathrm{T}} \cdot \dot{\boldsymbol{x}}=0 .
$$

The resulting sliding dynamic equation is

$$
\dot{\boldsymbol{x}}=\frac{\boldsymbol{\pi}_{x m}^{\mathrm{T}} \dot{\boldsymbol{x}}_{j} \dot{\boldsymbol{x}}_{i}-\boldsymbol{\pi}_{x m}^{\mathrm{T}} \dot{\boldsymbol{x}}_{i} \dot{\boldsymbol{x}}_{j}}{\boldsymbol{\pi}_{x m}^{\mathrm{T}}\left(\dot{\boldsymbol{x}}_{j}-\dot{\boldsymbol{x}}_{i}\right)}=: \boldsymbol{f}_{g}(\boldsymbol{x}, \boldsymbol{u}) .
$$

The coherences between the discrete states $\boldsymbol{x}_{D i}$, $\boldsymbol{x}_{D j}$ and $\boldsymbol{x}_{D m}$ are illustrated in fig. 1 .

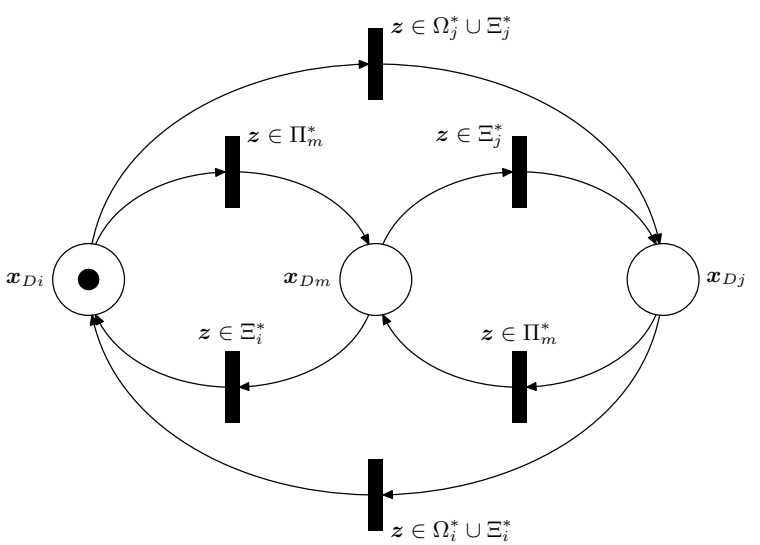

Fig. 1. Modelling of sliding modes

The transition sets as well as the sliding sets trigger a change of the discrete state and therefore do not belong to the neutral set of $\boldsymbol{x}_{D i}$. Hence, the neutral set has to be redefined:

$\Gamma^{\text {new }}\left(\boldsymbol{x}_{D i}\right):=\Gamma\left(\boldsymbol{x}_{D i}\right) \backslash\left(\bigcup_{j} \Xi_{j}^{*}\left(\boldsymbol{x}_{D i}\right) \cup \bigcup_{m} \Pi_{m}^{*}\left(\boldsymbol{x}_{D i}\right)\right)$

The considerations in this section lead to the definition of a new class of hybrid systems:

Definition 2.2

A piecewise affine system with sliding modes is a dynamic system with the state equation 


$$
\dot{\boldsymbol{x}}(t)=\left\{\begin{array}{lc}
\boldsymbol{A}_{1} \boldsymbol{x}(t)+\boldsymbol{B}_{1} \boldsymbol{u}(t)+\boldsymbol{a}_{1} & \text { if } \boldsymbol{z} \in \Omega_{1} \\
\vdots & \vdots \\
\boldsymbol{A}_{K} \boldsymbol{x}(t)+\boldsymbol{B}_{K} \boldsymbol{u}(t)+\boldsymbol{a}_{K} & \text { if } \boldsymbol{z} \in \Omega_{K} \\
\boldsymbol{f}_{g 1}(\boldsymbol{x}(t), \boldsymbol{u}(t)) & \text { if } \boldsymbol{z} \in \Pi_{1} \\
\vdots & \vdots \\
\boldsymbol{f}_{g S}(\boldsymbol{x}(t), \boldsymbol{u}(t)) & \text { if } \boldsymbol{z} \in \Pi_{S} .
\end{array}\right.
$$

The functions $\boldsymbol{f}_{g i}, i=1, \ldots, S$ are defined in eq. (4).

In the sequel it is assumed that no accumulation points of event times occur. This includes the wellposedness of piecewise affine systems according to (Schaft, A. van der and Imura J.-i., 2000) and excludes Zeno trajectories.

\section{THE DISCRETIZATION METHOD}

In order to deduce a difference equation which describes the discrete-time dynamic from a given continuous-time model of a piecewise affine system with sliding modes, it is useful to investigate the trajectories of the system. In the first subsection trajectories are calculated disregarding events. Later, events are taken into account and the problem is considered in which subsets of the extended state-space a consistent description of the discrete-time dynamic can be found. It is also discussed how the time when an event occurs may be determined.

\subsection{Trajectory functions}

For the calculation of trajectories the term trajectory function is defined (Hodrus, T. E. et al., 2004).

\section{Definition 3.1 (Trajectory function)}

The trajectory function

$$
\boldsymbol{x}\left(t_{0}+\tau\right)=\boldsymbol{\rho}\left(\boldsymbol{x}\left(t_{0}\right), \boldsymbol{u}\left(t_{0}\right), \tau\right)
$$

maps the state $\boldsymbol{x}\left(t_{0}\right)$ of a piecewise affine system to the state $\boldsymbol{x}\left(t_{0}+\tau\right)$ assuming that the system dynamic does not change in $\left[t_{0}, t_{0}+\tau\right]$ and that the input vector $\boldsymbol{u}(t)=\boldsymbol{u}\left(t_{0}\right)=$ const in this interval.

If the dynamic in the considered discrete state is affine the trajectory function is given by

$$
\begin{aligned}
& \boldsymbol{\rho}_{i}\left(\boldsymbol{x}\left(t_{0}\right),\right.\left.\boldsymbol{u}\left(t_{0}\right), \tau\right) \\
&=\boldsymbol{\Phi}(\tau) \boldsymbol{x}\left(t_{0}\right)+\boldsymbol{\Delta}(\tau)\left(\boldsymbol{B u}\left(t_{0}\right)+\boldsymbol{a}\right) \\
&=\boldsymbol{\Phi}(\tau) \boldsymbol{x}\left(t_{0}\right)+\boldsymbol{H}(\tau) \boldsymbol{u}\left(t_{0}\right)+\boldsymbol{\varphi}(\tau)
\end{aligned}
$$

with

$$
\boldsymbol{\Phi}(\tau)=\mathrm{e}^{\boldsymbol{A} \tau} \text { and } \boldsymbol{\Delta}(\tau)=\int_{\nu=0}^{\tau} \mathrm{e}^{\boldsymbol{A} \nu} d \nu .
$$

The dynamics in sliding modes are nonlinear and the trajectory function is hard to calculate. Approaches which can be found in the literature e. g. the TAYLOR-LIE-Series expansion or the method described in (Hohmann, S., 2003) often result in extremely complicated expressions.

A simple approach is to use the TAYLOR-Series expansion to linearize the dynamics with respect to the operating point $\boldsymbol{z}\left(t_{0}\right)$ and to assume this approximation to be valid in $\left[t_{0}, t_{0}+\tau\right]$ :

$$
\begin{aligned}
\dot{\boldsymbol{x}} & =\left.\frac{\partial \boldsymbol{f}_{g}}{\partial \boldsymbol{x}}\right|_{\boldsymbol{z}=\boldsymbol{z}\left(t_{0}\right)} \cdot\left(\boldsymbol{x}-\boldsymbol{x}\left(t_{0}\right)\right)+\boldsymbol{f}_{g}\left(\boldsymbol{x}\left(t_{0}\right), \boldsymbol{u}\left(t_{0}\right)\right) \\
& =\boldsymbol{A}_{g}\left(\boldsymbol{z}\left(t_{0}\right)\right) \cdot \boldsymbol{x}+\boldsymbol{a}_{g}\left(\boldsymbol{z}\left(t_{0}\right)\right)
\end{aligned}
$$

The derivative of $\boldsymbol{f}_{g}$ can be calculated analytically. The dynamic in eq. (6) is affine, so the trajectory function can be obtained as described above:

$$
\begin{aligned}
\boldsymbol{\rho}_{m}\left(\boldsymbol{x}\left(t_{0}\right), \boldsymbol{u}\left(t_{0}\right), \tau\right)=\mathrm{e}^{\boldsymbol{A}_{g}\left(\boldsymbol{z}\left(t_{0}\right)\right) \tau} \\
+\int_{\nu=0}^{\tau} \mathrm{e}^{\boldsymbol{A}_{g}\left(\boldsymbol{z}\left(t_{0}\right)\right) \nu} d \nu \cdot \boldsymbol{a}_{g}\left(\boldsymbol{z}\left(t_{0}\right)\right)
\end{aligned}
$$

Furthermore it meets the sliding condition (3). This method leads to good results as long as the interval length $\tau$ is not too large.

\subsection{Homogeneous sets}

In the sequel, $T$ denotes the sampling time.

The discretization method shall result in a difference equation

$$
\begin{aligned}
\boldsymbol{x}_{k+1} & =\left\{\begin{array}{cc}
\boldsymbol{g}_{1}\left(\boldsymbol{x}_{k}, \boldsymbol{u}_{k}\right) & \text { if } \boldsymbol{z}_{k} \in \Theta_{i_{1}^{(0)},[i]_{1}} \\
\vdots & \vdots \\
\boldsymbol{g}_{N}\left(\boldsymbol{x}_{k}, \boldsymbol{u}_{k}\right) & \text { if } \boldsymbol{z}_{k} \in \Theta_{i_{N}^{(0)},[i]_{N}}
\end{array}\right. \\
= & \boldsymbol{g}\left(\boldsymbol{x}_{k}, \boldsymbol{u}_{k}\right) .
\end{aligned}
$$

In the homogeneous sets $\Theta_{i(0),[i]} \subseteq \mathcal{Z}$ the dynamic of the discrete-time system can be described consistently. ${ }^{1}$

\section{Definition 3.2 (Homogeneous set)}

$A$ homogeneous set $\Theta_{i^{(0)},[i]}=\Theta_{i^{(0)}, i^{(1)}, \ldots, i^{(n)}}$ has got the following properties:

(1) $n=0$ : If $\boldsymbol{z}(k T) \in \Theta_{i(0)}$ holds, the system is in the discrete state $x_{D i(0)}$ and no change of the discrete state will occur in the interval $[k T,(k+1) T]$.

(2) $n>0$ : If $\boldsymbol{z}(k T) \in \Theta_{i^{(0)}, \ldots, i^{(n)}}$ holds, the system is in the discrete state $x_{D i(0)}$ and $n$ changes of the discrete state will occur in the interval $[k T,(k+1) T]$. The sequence of discrete states is $\boldsymbol{x}_{D i^{(1)}}, \ldots, \boldsymbol{x}_{D i^{(n)}}$.

1 In eq. (8) $i_{1}^{(0)},[i]_{1}, \ldots, i_{N}^{(0)},[i]_{N}$ denote the indices of different initial states $\boldsymbol{x}_{D i}(0)$ and sequences of states $\boldsymbol{x}_{D i}(1), \ldots, \boldsymbol{x}_{D i(n)}$. 
In the sequel $\boldsymbol{\rho}_{i}$ denotes the trajectory function of the discrete state $\boldsymbol{x}_{D i}$. If the homogeneous sets of the considered system are known, the difference equations $\boldsymbol{g}_{n}$ can be calculated. For this purpose the trajectory of the considered piecewise affine system is examined for $t \in[k T,(k+1) T]$ :

(1) $\boldsymbol{z}_{k} \in \Theta_{i(0)}$ : No event will occur within the next sampling interval. The difference equation is: $\boldsymbol{x}_{k+1}=\boldsymbol{\rho}_{i(0)}\left(\boldsymbol{x}_{k}, \boldsymbol{u}_{k}, T\right)$. The discrete dynamic is affine if $\boldsymbol{x}_{D i^{(0)}}$ is assigned to a trigger set (cf. eq. (5)) and nonlinear if $\boldsymbol{x}_{D i^{(0)}}$ is assigned to a sliding set (cf. eq. (7)).

(2) $\boldsymbol{z}_{k} \in \Theta_{i^{(0)}{ }_{i}(1)}$ : At a time $t+\tau^{*(1)}$ the trajectory intersects an active hyperplane $E^{*}$. This hyperplane is a superset of the transition set $\Xi^{*}$ or sliding set $\Pi^{*}$ which causes the change to the discrete state $\boldsymbol{x}_{D i(1)}{ }^{2}$. The intersection point is $\boldsymbol{x}^{*(1)}=\boldsymbol{\rho}_{i(0)}\left(\boldsymbol{x}_{k}, \boldsymbol{u}_{k}, \tau^{*(1)}\right){ }^{3}$

After the event the new dynamic is valid until $t=(k+1) T: \boldsymbol{x}_{k+1}=$ $\boldsymbol{\rho}_{i^{(1)}}\left(\boldsymbol{x}^{*(1)}, \boldsymbol{u}_{k}, T-\tau^{*(1)}\right)=\boldsymbol{g}_{n}\left(\boldsymbol{x}_{k}, \boldsymbol{u}_{k}\right)$. The resulting discrete dynamic is nonlinear.

This procedure can be easily extended to $n=$ $2,3, \ldots$ events in the interval $[k T,(k+1) T]$.

Only in special cases the boundaries of the homogeneous sets can be calculated analytically (Hodrus, T. E. et al., 2004). In order to determine the boundaries numerically the extended state space $\mathcal{Z}$ must be discretized. Then, for each discrete extended state $\boldsymbol{z}_{0}$ the assigned homogeneous set has to be determined. To solve this problem, a trajectory starting from $\boldsymbol{z}_{0}$ at $t=0$ has to be examined for $t \in[0, T]$. A sufficient criterion to find out whether an event occurs in the considered time interval is that the extended states $\boldsymbol{z}(0)=\boldsymbol{z}_{0}$ and $\boldsymbol{z}(T)$ are on different sides of one or several active planes. If this criterion is met, the time of intersection $\tau_{l}^{*(1)}$ has to be estimated for each intersected hyperplane $E_{l}^{*}$ (see section 3.3). The relevant intersection point $\boldsymbol{z}^{*(1)}$ is reached at $\tau^{*(1)}:=\min _{l} \tau_{l}^{*(1)}$. Then, the remaining trajectory starting from the intersection point $\boldsymbol{z}^{*(1)}$ has to be examined for $t \in\left[\tau^{*(1)}, T\right]$, etc. The affiliation of the investigated extended state $\boldsymbol{z}_{0}$ to a homogeneous set is determined when no further events occur.

The algorithm (as well as the estimation of the time of intersection) will fail if there are several intersection points with one active hyperplane in the examined time interval. Hence, it might be useful to take into account auxiliary time instances $t_{k}$ in the interval. If the sufficient criterion for an intersection is met in the interval $\left[t_{k_{0}}, t_{k_{0}+1}\right]$ of the length $\Delta t \leq T / N_{\tau}, N_{\tau}>1$ the risk of failure is

\footnotetext{
2 If the system is in a sliding state the active hyperplanes are determined by the condition of transition eq. (1).

3 The estimation of $\tau^{*(1)}$ is highlighted in section 3.3.
}

reduced and the estimation of the time of intersection will be more accurate. However, computation time will rise.

To prevent the estimation algorithm from converging to a wrong intersection point it makes sense to use the same auxiliary time instances $t_{k}$ for the calculation of the functions $\boldsymbol{g}_{n}$. The estimated time of intersection is influenced by the mean value $1 / 2 \cdot\left(t_{k_{0}}+t_{k_{0}+1}\right)$. Thus, the functions $\boldsymbol{g}_{n}$ are not consistent in $\Theta$ any longer but only in subsets $\Sigma$ of the homogeneous sets:

$$
\begin{aligned}
& \boldsymbol{g}_{n}\left(\boldsymbol{x}_{k}, \boldsymbol{u}_{k}\right) \\
& =\left\{\begin{array}{cc}
\boldsymbol{\gamma}_{n}^{1}\left(\boldsymbol{x}_{k}, \boldsymbol{u}_{k}\right) & \text { if } \boldsymbol{z}_{k} \in \Sigma_{\left[k_{0}\right]_{1}}\left(\Theta_{i_{n}^{(0)},[i]_{n}}\right) \\
\vdots & \vdots \\
\gamma_{n}^{M_{n}}\left(\boldsymbol{x}_{k}, \boldsymbol{u}_{k}\right) & \text { if } \boldsymbol{z}_{k} \in \Sigma_{\left[k_{0}\right]_{M_{n}}}\left(\Theta_{i_{n}^{(0)},[i]_{n}}\right)
\end{array}\right.
\end{aligned}
$$

Here, $\left[k_{0}\right]$ denotes the sequence of indices of the auxiliary time instances so that $t_{k_{0}^{(j)}}<\tau^{*(j)} \leq$ $t_{k_{0}^{(j)}+1}$ holds. That means that the homogeneous sets $\Theta$ are subdivided into sigma sets $\Sigma$ if auxiliary time instances are used.

\subsection{Estimation of the time of intersection}

In order to determine the time of intersection with a hyperplane the least solution $\tau^{*}$ of the equation

$$
f(\tau)=\boldsymbol{\omega}_{x}^{\mathrm{T}} \cdot \boldsymbol{\rho}\left(\boldsymbol{x}_{0}, \boldsymbol{u}_{0}, \tau\right)+\boldsymbol{\omega}_{u}^{\mathrm{T}} \boldsymbol{u}_{0}-\omega_{0}=0
$$

has to be found. For affine dynamics as well as sliding dynamics the (approximated) trajectory function can be written as

$$
\boldsymbol{\rho}\left(\boldsymbol{x}_{0}, \boldsymbol{u}_{0}, \tau\right)=\boldsymbol{\Phi}(\tau) \boldsymbol{x}_{0}+\boldsymbol{H}(\tau) \boldsymbol{u}_{0}+\boldsymbol{\varphi}(\tau)
$$

and the state equation as

$$
\dot{\boldsymbol{x}}=\boldsymbol{A} \boldsymbol{x}+\boldsymbol{B u}+\boldsymbol{a} .
$$

Hence, eq. (9) yields:

$$
\begin{aligned}
& f(\tau) \\
& =\boldsymbol{\omega}_{x}^{\mathrm{T}}\left(\boldsymbol{\Phi}(\tau) \boldsymbol{x}_{0}+\boldsymbol{H}(\tau) \boldsymbol{u}_{0}+\boldsymbol{\varphi}(\tau)\right)+\boldsymbol{\omega}_{u}^{\mathrm{T}} \boldsymbol{u}_{0}-\omega_{0} \\
& =\boldsymbol{\omega}_{x}^{\mathrm{T}} \boldsymbol{\Phi}(\tau) \boldsymbol{x}_{0}+\boldsymbol{\omega}_{x}^{\mathrm{T}} \boldsymbol{\Delta}(\tau)\left(\boldsymbol{B} \boldsymbol{u}_{0}+\boldsymbol{a}\right)+\boldsymbol{\omega}_{u}^{\mathrm{T}} \boldsymbol{u}_{0}-\omega_{0}
\end{aligned}
$$

If we assume $\boldsymbol{A}$ to be regular and diagonalizable $f(\tau)$ can be transformed into

$$
f(\tau)=\sum_{i=1}^{n_{x}} \eta_{i} \cdot \mathrm{e}^{\lambda_{i}}-\eta_{0}
$$

with

$$
\begin{aligned}
\eta_{i} & =\boldsymbol{w}_{i}^{\mathrm{T}}\left(\boldsymbol{x}_{0} \boldsymbol{\omega}_{x}^{\mathrm{T}}+\left(\boldsymbol{B} \boldsymbol{u}_{0}+\boldsymbol{a}\right) \boldsymbol{\omega}_{x}^{\mathrm{T}} \boldsymbol{A}^{-1}\right) \boldsymbol{v}_{i} \\
\eta_{0} & =\boldsymbol{\omega}_{x}^{\mathrm{T}} \boldsymbol{A}^{-1}\left(\boldsymbol{B} \boldsymbol{u}_{0}+\boldsymbol{A}\right)-\boldsymbol{\omega}_{u}^{\mathrm{T}} \boldsymbol{u}_{0}+\omega_{0} .
\end{aligned}
$$

In these equations $n_{x}$ denotes the dimension of the state space; $\lambda_{i}$ are the eigenvalues of $\boldsymbol{A}, \boldsymbol{v}_{i}$ are the right eigenvectors of $\boldsymbol{A}$ and $\boldsymbol{w}_{i}^{\mathrm{T}}$ are the left eigenvectors of $\boldsymbol{A}$. 
Eq. (10) shows that in general the time of intersection $\tau^{*}$ cannot be calculated analytically. Using the a priori knowledge $\tau^{*} \in\left[t_{k_{0}}, t_{k_{0}+1}\right]$ NEWTON's method can be applied to estimate $\tau^{*}$. In order to keep the difference equations for the time discrete system as simple as possible only one iteration is performed. With the initial value $\bar{\tau}=1 / 2\left(t_{k_{0}}+t_{k_{0}+1}\right)$ the result of the estimation is:

Using

$$
\hat{\tau}^{*}=\bar{\tau}-\frac{f(\bar{\tau})}{f^{\prime}(\bar{\tau})}
$$

$$
\frac{d}{d \tau} \boldsymbol{\Phi}(\tau)=\boldsymbol{A} \boldsymbol{\Phi}(\tau)=\boldsymbol{\Phi}(\tau) \boldsymbol{A}
$$

and

$$
\frac{d}{d \tau} \boldsymbol{\Delta}(\tau)=\boldsymbol{\Phi}(\tau)
$$

the derivative of $f(\tau)$ yields:

$$
f^{\prime}(\tau)=\boldsymbol{\omega}_{x}^{\mathrm{T}} \boldsymbol{\Phi}(\tau)\left(\boldsymbol{A} \boldsymbol{x}_{0}+\boldsymbol{B} \boldsymbol{u}_{0}+\boldsymbol{a}\right)
$$

Assuming $t_{k_{0}+1}-t_{k_{0}} \leq T / N_{\tau}$ the estimation is good if $\left|\tau^{*}-\hat{\tau}^{*}\right| \ll T / N_{\tau}$. With eq. (10) it can be proved for a regular and diagonalizable matrix $\boldsymbol{A}$ that this condition is met if

$$
\frac{T}{N_{\tau}} \ll \frac{1}{\max _{i}\left|\lambda_{i}\right|}
$$

holds. This inequality can be used to choose an adequate $N_{\tau}$ for each discrete state.

To handle sliding modes correctly the estimated intersection point $\hat{\boldsymbol{x}}^{*}$ must meet the condition

$$
\boldsymbol{\omega}_{x}^{\mathrm{T}} \boldsymbol{x}^{*}+\boldsymbol{\omega}_{u}^{\mathrm{T}} \boldsymbol{u}_{0}-\omega_{0}=0
$$

exactly. Hence, $\boldsymbol{x}\left(\hat{\tau}^{*}\right)$ has to be projected onto the hyperplane. A convenient direction of the projection is the direction of the tangent of the trajectory at $\hat{\tau}^{*}: \dot{\boldsymbol{x}}\left(\hat{\tau}^{*}\right)=\boldsymbol{A} \boldsymbol{x}\left(\hat{\tau}^{*}\right)+\boldsymbol{B} \boldsymbol{u}_{0}+\boldsymbol{a}$.

This yields:

$\hat{\boldsymbol{x}}^{*} \approx\left(\boldsymbol{I}-\frac{\dot{\boldsymbol{x}}\left(\hat{\tau}^{*}\right) \boldsymbol{\omega}_{x}^{\mathrm{T}}}{\boldsymbol{\omega}_{x}^{\mathrm{T}} \dot{\boldsymbol{x}}\left(\hat{\tau}^{*}\right)}\right) \boldsymbol{x}\left(\hat{\tau}^{*}\right)-\frac{\left(\boldsymbol{\omega}_{u}^{\mathrm{T}} \boldsymbol{u}_{0}-\omega_{0}\right) \dot{\boldsymbol{x}}\left(\hat{\tau}^{*}\right)}{\boldsymbol{\omega}_{x}^{\mathrm{T}} \dot{\boldsymbol{x}}\left(\hat{\tau}^{*}\right)}$

\section{EXAMPLE}

As an example the following autonomous system is analyzed:

$$
\dot{\boldsymbol{x}}(t)=\left\{\begin{array}{l}
{\left[\begin{array}{rr}
0 & 0 \\
-2 & 0
\end{array}\right] \boldsymbol{x}(t)+\left[\begin{array}{l}
2 \\
3
\end{array}\right] \quad \text { if } \boldsymbol{z}(t) \in \Omega_{1}} \\
\frac{2}{3}\left[\begin{array}{rr}
-1 & -1 \\
1 & 1
\end{array}\right] \boldsymbol{x}(t)+\left[\begin{array}{l}
4 \\
4
\end{array}\right] \quad \text { if } \boldsymbol{z}(t) \in \Omega_{2} \\
\frac{1}{3}\left[\begin{array}{rr}
-2 & 1 \\
-2 & 1
\end{array}\right] \boldsymbol{x}(t)+\frac{1}{3}\left[\begin{array}{l}
1 \\
2
\end{array}\right] \quad \text { if } \boldsymbol{z}(t) \in \Omega_{3}
\end{array}\right.
$$

with

$$
\Omega_{1}=\left\{\boldsymbol{x} \mid\left[\begin{array}{ll}
-2.5 & 1
\end{array}\right] \boldsymbol{x}+2.5>0\right\},
$$

$$
\begin{aligned}
& \Omega_{2}=\left\{\boldsymbol{x} \mid\left[\begin{array}{rr}
2.5 & -1 \\
-4 & 1
\end{array}\right] \boldsymbol{x}-\left[\begin{array}{r}
2.5 \\
-12
\end{array}\right]>\mathbf{0}\right\} \text { and } \\
& \Omega_{3}=\{\boldsymbol{x} \mid[4-1] \boldsymbol{x}-12>0\} .
\end{aligned}
$$

The state variables are limited to $0 \leq x_{1} \leq 3.5$ and $-2 \leq x_{2} \leq 2$. The evaluation of the condition of transition (1) yields:

(1) If $\boldsymbol{x} \in \partial \Omega_{1} \cap \partial \Omega_{2}$ the system will change to the discrete state $\boldsymbol{x}_{D 2} . \quad \Rightarrow \Xi_{2}=\partial \Omega_{1} \cap \partial \Omega_{2}$

(2) If $\boldsymbol{x} \in \partial \Omega_{2} \cap \partial \Omega_{3}$ the condition is met for both directions: The system will change into a sliding state $\boldsymbol{x}_{D 4} . \quad \Rightarrow \Pi_{4}=\partial \Omega_{2} \cap \partial \Omega_{3}$

The continuous trajectory in fig. 2(a) demonstrates the transitions from $\Omega_{1}$ to $\Omega_{2}$ and from $\Omega_{2}$ to $\Pi_{4} \cdot{ }^{4}$

The system is discretized with the sample time $T=0.4 \mathrm{~s}$. First the conventional discretization method is applied. That means, the differential equation for $\Omega_{i}$ is given by

$$
\boldsymbol{x}_{k+1}=\boldsymbol{\Phi}(T) \boldsymbol{x}_{k}+\boldsymbol{\varphi}(T) \text {. }
$$

Fig. 2(a) and 2(b) show simulation results. After the first transition obvious discrepancies can be observed. After the transition to $\Omega_{3}$ the discretetime system toggles between the states $\boldsymbol{x}_{D 3}$ and $\boldsymbol{x}_{D 2}$.

Applying the new method shows that the discretetime affine dynamics eq. (11) are only valid in the homogeneous sets $\Theta_{i} \subset \Omega_{i}$. Furthermore in the considered section of the state-space there is a homogeneous set $\Theta_{4}$ which is identical to the sliding set $\Pi_{4}$ as well as four more homogeneous sets with nonlinear dynamics. If the extended state $\boldsymbol{z}_{k}$ is in one of these sets, one $\left(\Theta_{1,2}, \Theta_{2,4}, \Theta_{3,4}\right)$ or even two $\left(\Theta_{1,2,4}\right)$ transitions will take place within the next sampling period. The simulation results in fig. 2(a) and 2(b) demonstrate the high accuracy of the discrete-time model.

Fig. 3(a) and 3(b) show the nonlinear $x_{1, k+1^{-}}$and the $x_{2, k+1}$-map of the discrete-time system. The black lines represent the boundaries of the trigger sets. The affine discrete dynamic which is valid in the homogeneous sets $\Theta_{1}, \Theta_{2}$ and $\Theta_{3}$ is represented by subsets of planes. If the conventional discretization method is applied, these planes are extended to the whole trigger set.

\section{CONCLUSIONS}

The presented method maps a continuous-time piecewise affine system to a discrete-time piecewise defined nonlinear system which reproduces the dynamic of the original system precisely. In order to obtain a discrete-time piecewise affine system the occurring nonlinear dynamics have to

4 The different shades of grey represent the trigger sets. 


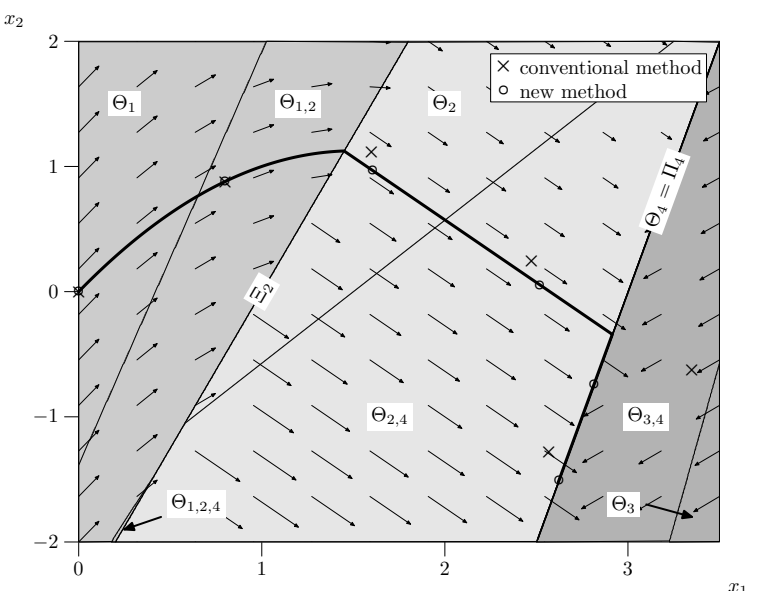

(a) Homogeneous sets of the piecewise affine system
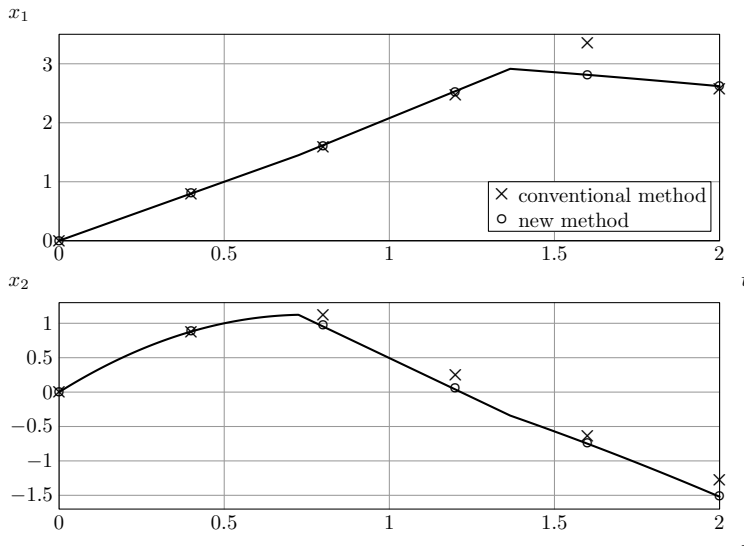

(b) Trajectory of the piecewise affine system

Fig. 2.

be approximated by piecewise affine dynamics. With a more general definition of homogeneous sets the method can also be applied to systems with jumps, discrete inputs and non-disjunctive trigger sets.

The disadvantages of this approach are the high complexity of the resulting model and the effort to determine the boundaries of the homogeneous sets. Here, a more effective algorithm has to be found.

\section{REFERENCES}

Bemporad, A. and Morari, M. (1999). Control of systems integrating logic, dynamics, and contraints. Automatica 35, 407-427.

Branicky, M. S. (1995). Studies in Hybrid Systems: Modeling, Analysis, and Control. PhD thesis. MIT.

Hodrus, T. E., Schwarz, M. and Krebs, V. (2004). A Time Discretization Method for a Class of Hybrid Systems. Proceedings of NOLCOS, IFAC Symposium on Nonlinear Control Systems.

Hohmann, S. (2003). Entwurf nichtlinearer Abtastregelungen auf der Basis einer polynomialen

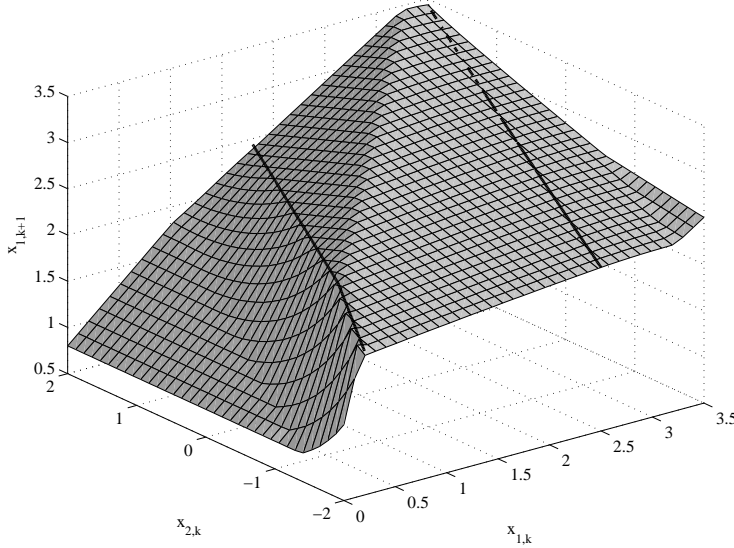

(a) $x_{1, k+1}$-map

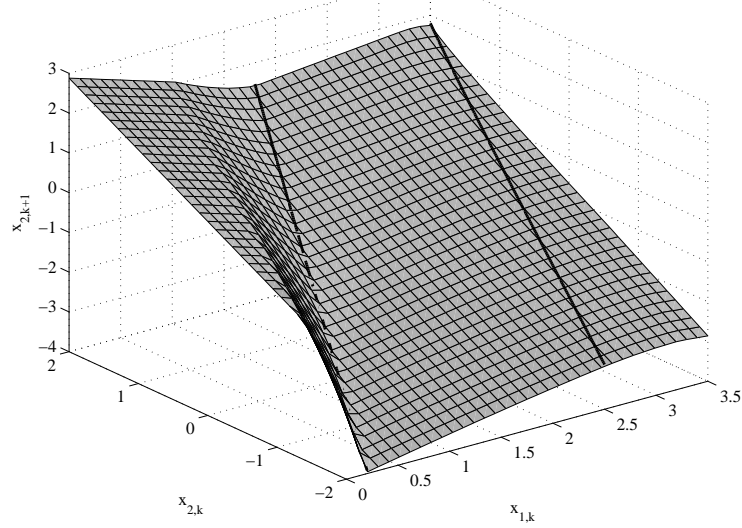

(b) $x_{2, k+1}$-map

Fig. 3.

Zeitdiskretisierung. In: Fortschritt-Berichte VDI, Reihe 8, Nr. 986. VDI Verlag GmbH. Düsseldorf.

Münz, E. and Krebs, V. (2002). Identification of Hybrid Systems Using a Priori Knowledge. In: Proceedings of the IFAC World Congress. Barcelona, Spain. p. CD.

Nenninger, G. M. (2001). Modellbildung und Analyse hybrider dynamischer systeme als Grundlage für den Entwurf hybrider Steuerungen. In: Fortschritt-Berichte VDI, Reihe 8, Nr. 902. VDI Verlag GmbH. Düsseldorf.

Schaft, A. van der and Imura J.-i. (2000). Characterization of Well-Posedness of PiecewiseLinear Systems. IEEE Transactions on Automatic Control 45(9), p. 1600-1619.

Schnabel, M. (2001). Diskret-kontinuierliche Systeme: Optimale Steuerung und Beobachtung. In: Fortschritt-Berichte VDI, Reihe 8, Nr. 900. VDI Verlag GmbH. Düsseldorf.

Utkin, V. I. (1992). Sliding Modes in Control and Optimization. Springer-Verlag. Berlin. 